This item was submitted to Loughborough's Research Repository by the author.

Items in Figshare are protected by copyright, with all rights reserved, unless otherwise indicated.

\title{
Underexpanded jet development from a rectangular nozzle with aft-deck
}

PLEASE CITE THE PUBLISHED VERSION

http://dx.doi.org/10.2514/1.J053376

PUBLISHER

(c) American Institute of Aeronautics and Astronautics

\section{VERSION}

AM (Accepted Manuscript)

\section{PUBLISHER STATEMENT}

This work is made available according to the conditions of the Creative Commons Attribution-NonCommercialNoDerivatives 4.0 International (CC BY-NC-ND 4.0) licence. Full details of this licence are available at: https://creativecommons.org/licenses/by-nc-nd/4.0/

\section{LICENCE}

CC BY-NC-ND 4.0

\section{REPOSITORY RECORD}

Behrouzi, Parviz, and Jim McGuirk. 2014. "Underexpanded Jet Development from a Rectangular Nozzle with Aft-deck”. Loughborough University. https://hdl.handle.net/2134/18101. 


\title{
Underexpanded Jet Development from a Rectangular Nozzle with Aft-Deck
}

\author{
Parviz Behrouzi ${ }^{*}$ and James J. McGuirk ${ }^{\dagger}$ \\ Department of Aeronautical and Automotive Engineering, Loughborough University, \\ Loughborough, Leicestershire, LE11 3TU, UK
}

An experimental study is reported of underexpanded supersonic jet plumes issuing from a high aspect ratio convergent rectangular nozzle. Schlieren visualization, Pitot probe, and LDA measurements are carried out to capture the plume development in the near-field, and in particular the effect on the plume flow of a finitelength extended shelf or aft-deck attached to the lower nozzle wall. This creates asymmetry in the inviscid shock cell pattern, and the entrainment characteristics, both of which influence shear layer growth and plume trajectory. A net pressure force is induced on the aft-deck wall, which leads to transverse deflection of the jet plume once it leaves the aft-deck - both upwards and downwards depending on aft-deck length and NPR. For sufficiently high NPR and a sufficiently long aft-deck, separation and reattachment of the plume from the aft-deck is observed. Detailed mapping of both mean velocity and turbulence in the plume near-field has been carried out, enabling comparison of flow behavior for a clean nozzle and a nozzle with aft-deck. The data provided are proposed as a suitable benchmark validation test case for CFD studies of rectangular nozzle plumes with aft-deck interaction effects.

\section{Nomenclature}

$$
\begin{array}{ll}
A R & =\text { Nozzle aspect ratio }\left(\mathrm{W}_{\mathrm{n}} / \mathrm{H}_{\mathrm{n}}\right) \\
D_{h} & =\text { Nozzle exit hydraulic diameter }\left(=\left[4 * \mathrm{~W}_{\mathrm{n}} * \mathrm{H}_{\mathrm{n}}\right] /\left[2 *\left(\mathrm{~W}_{\mathrm{n}}+\mathrm{H}_{\mathrm{n}}\right)\right]\right. \\
H_{n} & =\text { Nozzle exit height (minor axis dimension) } \\
\mathrm{L} & =\text { Aft-deck length } \\
\mathrm{M}_{\mathrm{c}} & =\text { Convective Mach number of shear layer }
\end{array}
$$

\footnotetext{
* Research Associate, Department of Aeronautical and Automotive Engineering, Loughborough University, Loughborough, Leicestershire, LE11 3TU, UK, P.Behrouzi@lboro.ac.uk

${ }^{\dagger}$ Professor of Aerodynamics, Department of Aeronautical and Automotive Engineering, Loughborough University, Loughborough, Leicestershire, LE11 3TU, UK, J.J.McGuirk@lboro.ac.uk
} 


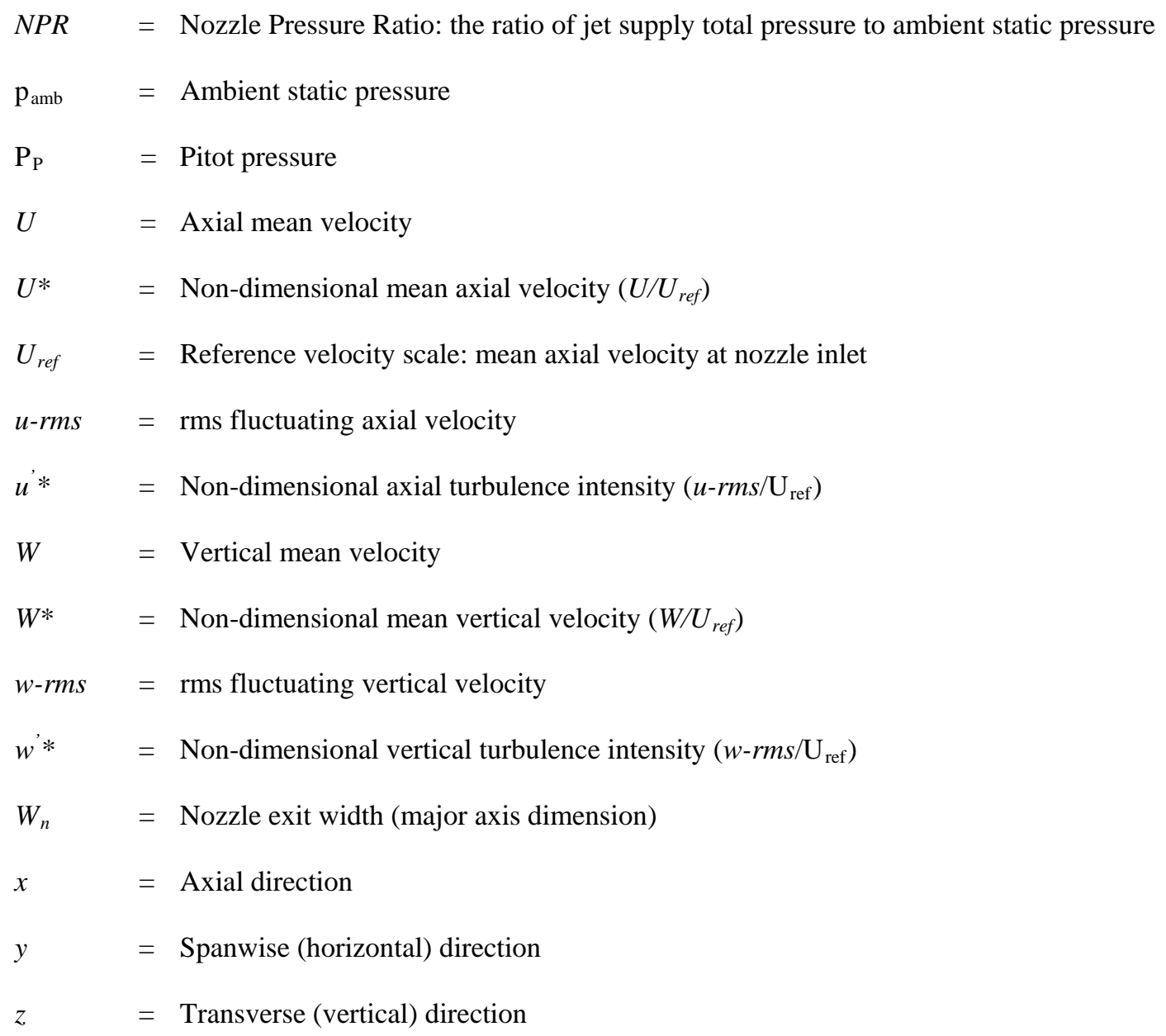

\section{Introduction}

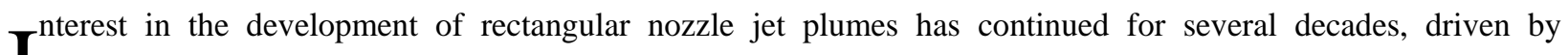
observations that rectangular nozzle jet/ambient mixing and velocity (and temperature for hot jets) decay rates are faster than for an axisymmetric nozzle. In civil aircraft propulsion plume mixing affects jet noise production, whereas in military applications the focus of interest is on reduced IR signature. The first studies into rectangular jet development were carried out some 45 years ago by Trentacoste and Sforza ${ }^{1}$ and later Sfeir ${ }^{2}$ using single hot-wire anemometry. These early low Mach No. studies showed the complexity of 3D jet flows; 'saddle-shaped' velocity profiles were measured in the major axis plane, as well as an 'axis-switching' effect. This latter is the exchange of 
orientation of major and minor axes of the jet plume from the original nozzle orientation as the plume develops downstream; very far downstream of course all nozzle shapes eventually transition into round plumes. McGuirk \& Rodi $^{3}$ predicted these measurements using a high Re k- $\varepsilon$ turbulence model and were able to calculate most of the observed features (including axis-switching, but not the saddle-shaped profiles). They noted that secondary flow conditions at the nozzle exit, determined by the internal shape of the supply duct feeding the nozzle, could play a dominant role in determining the occurrence and location of axis-switching via a strong vena-contracta effect. Sfeier's ${ }^{2}$ data confirmed this since the switch-over occurred earlier when the nozzle discharged from a sharp-edged orifice rather than a long constant area supply duct. Krothapalli et al. ${ }^{4}$ measured mean velocities and turbulent shear stresses in a $\mathrm{Re}=1.2 \times 10^{4}$ low subsonic rectangular jet plume; nozzles with Aspect Ratios (AR) between 5.5 and 16.7 were tested. The results showed that at high AR the flowfield could be characterized by the presence of three distinct regions: potential core, essentially 2D (plane) jet behavior, and 2D (axisymmetric) jet behavior. For AR less than 10 (typical of practical applications), no 2D plane jet region was identified and the initial decay rate depended on nozzle geometry. It was suggested that the appearance of saddle-shaped major axis profiles might be related to 3D vortex rings shed from the nozzle lip.

The measurements of $\mathrm{Zaman}^{5}$ (mainly on a nozzle with $\mathrm{AR}=3$ at $\mathrm{Re}=4.5 \times 10^{5}$ and $\mathrm{M}=0.31$ ) provided strong evidence of this link between axis-switching and saddle-shaped profiles and the 3D development of coherent turbulent structures present in the form of non-axisymmetric azimuthal vortex rings originating at the nozzle lip. Most of the data were focused on the effect of streamwise-vortex-inducing tabs placed on the nozzle sides, or axial forcing/excitation of the jet to enhance mixing, but data were also taken for a 'clean' nozzle flow for comparison purposes. Based on earlier measurements for elliptic nozzles (Ho and Gutmark ${ }^{6}$, Hussain and Husain ${ }^{7}$ ), Zaman $^{5}$ explained how azimuthal vorticity (from the internal nozzle wall boundary layers), when rolled up at the nozzle lip into a vortex ring with a non-axisymmetric shape (determined by the nozzle cross-section), would deform as it convected downstream. This was due to the self-induced (Biot-Savart law) velocity, which varied around the ring due to the difference in local vorticity magnitude (varying momentum thickness of the wall boundary layer) and varying azimuthal curvature of the vortex tube. Segments of the vortex at either end of the major axis have higher induced velocity than segments at the ends of the minor axis. This leads to the fluid at the ends of the major axis moving faster downstream and radially inwards, with opposite effects occurring on the minor axis plane, causing the vortex to move out of plane. 
Such 3D deformations of the asymmetric vortex rings that are features of rectangular/elliptical nozzles have been established as the driving mechanism for axis-switching. The review of flow control in non-circular jets by Gutmark and Grinstein ${ }^{8}$ and the Large Eddy Simulation CFD predictions of Grinstein et al. ${ }^{9}$ and Grinstein ${ }^{10}$ provided further details in support of these ideas. Two important characteristics are that: (i) enhanced ambient entrainment via the deformation of large scale 3D vortex structures and (ii) increased small scale turbulence due to the breakdown of the large scale structures are the primary drivers of axis-switching and increased mixing. Depending on the strength of the azimuthal vortex tube distortion, streamwise vorticity can also be created, embedded within the azimuthal vorticity field. This is found to occur (in both experiments and CFD) in regions of high curvature such as the corners of a rectangular nozzle; this leads to the appearance of streamwise vorticity and is one cause of the saddle-shaped profile structure. All these descriptions and conclusions on the fluid mechanics of near-field rectangular jet development have been substantiated in a study by Lozanova and Stankov ${ }^{10}$, who conducted X-probe hot-wire measurements for nozzles with AR between 3 and 10, and provided detailed 2D mapping of mean vorticity as well as turbulent normal and shear stresses in the near-field for $A R=3$. The effect of nozzle AR on jet development was further investigated by Quinn ${ }^{11,12}$ and Mi et al. ${ }^{13}$; these results showed that near-field turbulence levels and mixing were increased as aspect ratio (AR) increased, up to AR 20.

The vast majority of the above studies were carried out for low speed jets. Only the work of Zaman ${ }^{5}$ extended experimental evidence to higher Mach numbers, where (intriguingly) it was found that the same AR=3 rectangular nozzle displayed little evidence of axis-switching at $\mathrm{M}=0.3$, but significant switch behavior at $\mathrm{M}=1.6$. The explanation for this, however, was the presence of a screech phenomenon observed in the supersonic jet. This would enhance the strength of the asymmetric azimuthal vortex rings mentioned above by effectively introducing an axial plane acoustic wave forcing of the jet velocity, thus reinforcing the mechanism for axis-switching that was too weak to be noticeable at the lower Mach number. This mechanism leads to enhanced mixing and a shorter potential core. Very little other work has examined possible compressibility effects at high subsonic or supersonic jet Mach numbers - according to the review of Gutmark and Grinstein ${ }^{8}$ this is principally the experiments of Krothapalli et al. ${ }^{15}$ and Gutmark et al. ${ }^{16}$. These studies observed two extra effects on jet mixing introduced by supersonic and improperly expanded jet conditions (in addition to the screech-induced axial forcing already mentioned). The first was the reduction in shear layer growth rate (i.e. the tendency for a longer potential core) when the convective Mach number $\mathrm{M}_{\mathrm{c}}$ of the shear layer was high. $\mathrm{M}_{\mathrm{c}}$ is a Mach number defined using the velocity difference across the shear 
layer, and the average speed of sound between the faster/slower sides of the shear layer: $M_{c}=\left(U_{1}-U_{2}\right) / a_{12}(U$ and a are the flow speed and speed of sound and $1 / 2$ indicates faster/slower streams). Papamoschou and Roshko ${ }^{17}$ first measured this effect in a 2D plane shear layer. Compressible effects are small until $\mathrm{M}_{\mathrm{c}} \sim 0.4$, but then lead to a halving of the low Mach number spreading rate between $\mathrm{M}_{\mathrm{c}}=0.4$ and 0.8. Barone, Oberkampf \& Blottner ${ }^{18}$ reviewed 11 sets of experiments studying this effect and provided a recommended curve for the collapse of the shear layer spreading rate of compressible planar shear layers. This is often used to calibrate compressibility effects in Reynolds Averaged Navier Stokes (RANS) turbulence models. The second compressible effect is the appearance of a compression/expansion wave shock cell pattern in the jet core for off-design Nozzle Pressure Ratio (NPR, i.e. underand over-expanded conditions) as the nozzle exit flow transitions to static pressure equilibrium with the ambient. At high NPR, the shock cell pattern can cause a barrel-shaped curvature to the outer edge of the jet core. The total pressure losses associated with the flow passing through the oblique shock waves also (even for axisymmetric jets, see Feng and McGuirk ${ }^{19}$ ) can cause the velocity near the centerline to be less than near the inviscid core edge, helping to reinforce any saddle-shaped profile caused by vortex ring deformation. It is thus obvious that compressibility effects are complex, and might also counteract each other. Further evidence for high Mach rectangular jets would be valuable.

Installed nozzles will often have at least one side where nearby solid surfaces restrict entrainment into the jet plume. This is often the result of a desire to achieve close integration of propulsion nozzle and aircraft afterbody; in some applications the shielding effect of such a surface may be used to advantage. The effect on jet development of extended walls attached to the short sides of a high aspect ratio rectangular nozzle has been studied by Deo et al. ${ }^{20}$ and also in papers by Alnahhal and Panidis ${ }^{21}$ and Alnahhal et al. ${ }^{22}$. The motivation behind these works was to examine how long sidewalls introduced on both sides of the nozzle or an endplate from which the nozzle emerged, would influence (and perhaps lengthen) the 2D planar region of the jet decay. Only large aspect ratio nozzles $(A R=15)$ were considered. Sideplates did extend the 2D region by improving the stability of the 2D roller eddies in the shear layer, delaying their development into 3D structures. An endplate also affected the flow, mainly by constraining the entrainment from behind the nozzle exit plane. Neither of these effects is of great significance in the near-field, low aspect ratio geometries of relevance here.

A more practically relevant motivation for examining the influence of a sidewall arises in military propulsion nozzle applications, where a single and finite-length sidewall may be present and used to shield the hot plume from 
view. Such geometry modifications, particularly for plumes discharging from nozzles at supercritical NPRs, will potentially also affect the internal nozzle performance, and this has been studied by Lamb et al. ${ }^{23}$, who referred to the wall geometry as an 'extended shelf'. Only wall static pressure measurements were presented in the paper by Lamb et al. ${ }^{23}$, so this work did little to provide any understanding of the reasons for the impact of an aft-deck on plume development. Deere et $\mathrm{al}^{24}$ carried out a computational investigation of a rectangular convergent-divergent nozzle using fluidic internal jet discharge to effect thrust vectoring of the exhaust plume; this work was the first to use the description 'aft-deck'. Avoidance of separation of the underexpanded jet from the aft-deck surface was one of the main areas of interest. Whilst the CFD predictions presented did show that aft-deck separation could occur, and its location could be manipulated using fluidic control, the numerical solutions were not compared to experiments, so the CFD approach used could not be properly validated for its ability to predict aft-deck effects. Since this work, no investigations, in particular experimental studies, have been reported on the impact of an aftdeck on the jet plume flowfield development. This is rather surprising, since proper understanding of this is important in nozzle design. Further, how accurately CFD can predict the basic plume and also any aft-deck effects on flowfield development are important questions. To answer these questions requires detailed, high quality (benchmark) experimental data for both mean and turbulence flow characteristics. For this reason, a measurement program to extend the database on high Mach rectangular free jets, and to examine the effect of an aft-deck on supersonic jet plume behavior is reported in this paper; production of a CFD validation database was one of the prime objectives of the study.

\section{Experimental Facility and Instrumentation}

\section{A. High Pressure Nozzle Test Facility (HPNTF).}

The measurements reported here were carried out in a specially designed and constructed high pressure air flow rig for nozzle flow studies. The Loughborough University High Pressure Nozzle Test Facility (HPNTF see Behrouzi et al. $^{25}$ and Behrouzi and McGuirk ${ }^{26}$ ) consists of two compressors, air storage tanks, delivery pipelines to the test cell, control valves, a combustion unit for heating the jet flow if needed, a rig control panel and rig instrumentation. The compressors draw in $\sim 0.9 \mathrm{~m}^{3} / \mathrm{s}$ of ambient air $(\sim 1 \mathrm{~kg} / \mathrm{sec})$ and supply high pressure air with a maximum pressure of $1.4 \times 10^{6}$ Pascal (gauge). The main air storage tank has a volume of $110 \mathrm{~m}^{3}$ and acts as a buffer to damp 
pressure fluctuations as well as providing an air supply for the HPNTF to be run in blowdown mode. A desiccanttype drier is used to achieve a dew-point of the high pressure air of $-40^{\circ} \mathrm{C}$. Two automatic control valves installed in series are employed to hold the nozzle mass flow rate constant. The facility can be used in continuous or blowdown mode. In blowdown mode (for NPR levels higher than $\sim 2.5$ for a typical nozzle size) the tanks are first filled to a substantially higher pressure than the NPR for the selected test operating point. The $1^{\text {st }}$ automatic control valve reduces the pressure level to $\sim 5 \times 10^{6}$ Pascal (gauge). The second control valve drops the pressure from this to the required nozzle operating pressure and holds this to within $+/-1 \%$ during the test run time. Typical blowdown run times are between 15- 30 minutes depending on NPR. Fig. 1 presents a photo of the rig, indicating its major components.

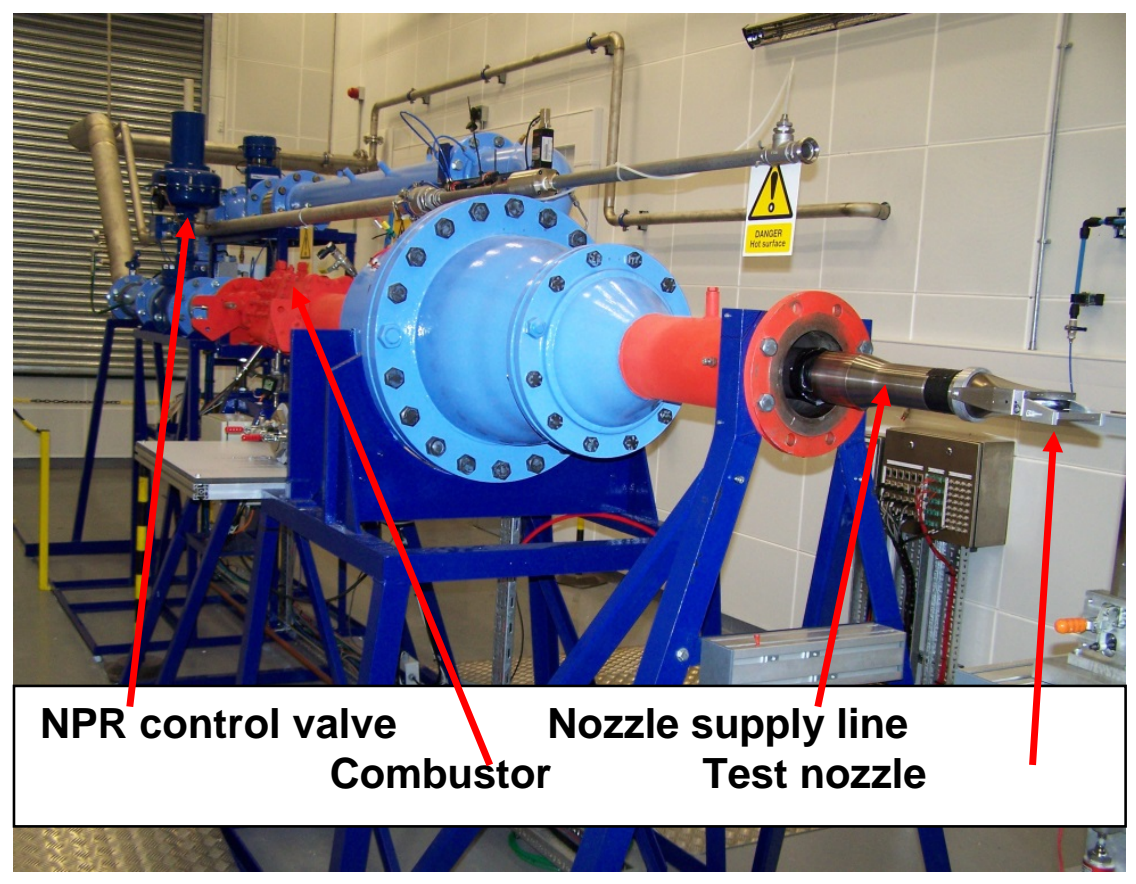

Fig. 1 Photo of the HPNTF with rectangular convergent nozzle installed 


\section{B. Rectangular Nozzle Design.}

The nozzle is a convergent rectangular nozzle with a round inlet section of $54 \mathrm{~mm}$ internal diameter (to fit the facility high pressure air supply line shown in Fig.1) and an exit rectangular geometry with major and minor axis dimensions of $93.8 \mathrm{~mm} \times 12.76 \mathrm{~mm}(\mathrm{AR}=7.35)$ and a trailing edge lip thickness of $0.5 \mathrm{~mm}$. The nozzle supply duct was a round pipe which contained a 4/1 contraction to reduce boundary layer thickness, followed by a $2 \mathrm{~m}$ length of straight pipe. This enables the boundary layer thickness just upstream of the nozzle to be estimated. The nozzle geometry consisted of a round to rectangular contraction of $4 / 1$ are ratio as shown in Fig. 2. The nozzle exit hydraulic diameter $\mathrm{D}_{\mathrm{h}}$ is $22.46 \mathrm{~mm}$ and in what follows this is used to make all length variables non-dimensional. Fig. 2 presents a schematic and a photo of the installed clean nozzle, indicating also the Cartesian coordinates used to report measurements below.
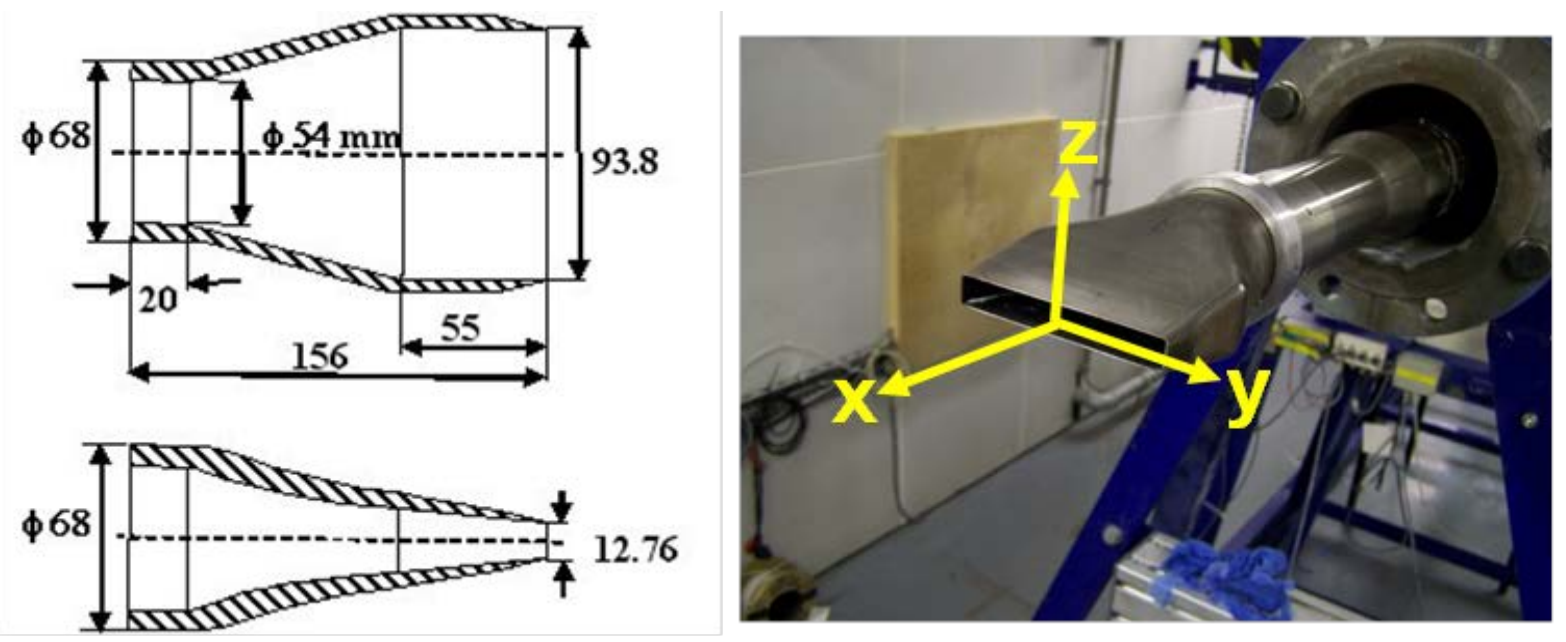

Fig. 2 Dimensions and photo of the convergent rectangular test nozzle

\section{Aft-deck Geometry.}

A special collar section was designed to hold a rectangular plate used as an aft-deck to the nozzle as shown schematically in Fig. 3. The datum aft-deck was sized at $53.3 \mathrm{~mm}$ long $\left(2.37 \mathrm{D}_{\mathrm{h}}\right)$ by $93.8 \mathrm{~mm}$ wide $\left(4.18 \mathrm{D}_{\mathrm{h}}\right)$. To test the effect of aft-deck size on plume development, two additional geometries were used with the same width but with 
$\sim 40 \%$ shorter $\left(30.5 \mathrm{~mm}, 1.36 \mathrm{D}_{\mathrm{h}}\right)$ and longer $\left(75.5 \mathrm{~mm}, 3.37 \mathrm{D}_{\mathrm{h}}\right)$ axial dimensions. Fabrication and attachment of the aft-deck was carried out such that steps between nozzle internal wall and extended shelf surface were avoided.

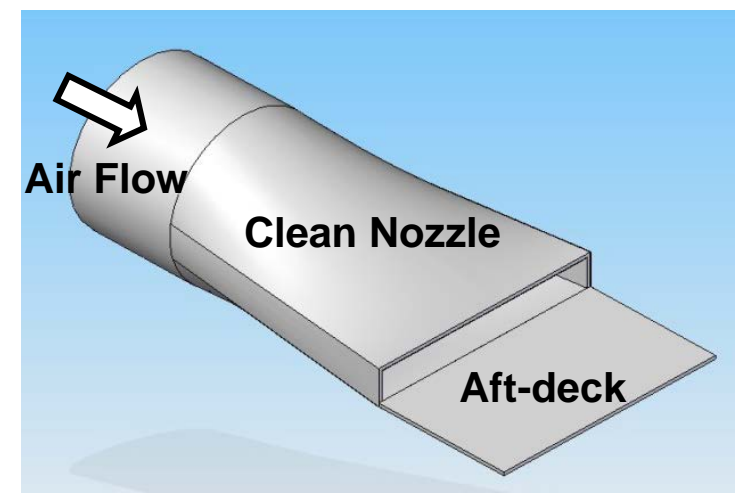

Fig. 3 Aft-deck attached to lower nozzle wall

\section{Instrumentation}

The Schlieren technique is a convenient method of visualizing the position and shape of shock waves. The Schlieren unit operated in the HPNTF is a Z-type design, consisting of a light source, two concave mirrors of 10" diameter, and two plane mirrors of 12" diameter, a rainbow filter unit and a Sony digital camera. Schlieren pictures were taken from top and side orientations of the jet plume, focusing on the region just downstream of nozzle exit.

A two component DANTEC LDA system was used in backscatter mode for non-intrusive measurement of timeaveraged velocity and turbulence statistics. This unit consisted of a 4W Argon-Ion laser source, an opto-electronic laser beam manipulator, an LDA optic head, an F80 signal processor and a 3D traversing table. A SCITEK-10F01 solid particle generator employing $\mathrm{Al}_{2} \mathrm{O}_{3}$ powder (de-agglomerated alumina powder of $0.3 \mu$ m median size) was used to seed the flow. The LDA system incorporated a beam expander unit (to provide a smaller LDA measuring volume) for velocity measurements. The measuring volume dimensions ( $\mathrm{x}, \mathrm{y}$, and $\mathrm{z}$ ) were $0.076 \mathrm{~mm}, 1.254 \mathrm{~mm}$ and $0.076 \mathrm{~mm}$ respectively. A combination of a validated sample number of 20,000 or 30 seconds sampling time (whichever was fulfilled first) was found suitable for capture of smooth statistics in the LDA measurements. The selected sampling time and number of samples was found to give repeatable LDA measurement results. No corrections were made for sampling bias in the LDA results. Any associated errors were minimized by using high data rates compared to typical velocity fluctuation levels. The signal processor was operated in trigger mode such that data were acquired at fixed time intervals; a typical data rate used was $2 \mathrm{KHz}$. The error magnitude for LDA probe positioning was estimated at $0.1 \mathrm{~mm}$. 
A simple Pitot pressure probe was used for measurement of the jet plume total pressure field. DANTEC MiniCTA data acquisition software was adapted for use with an NI-6023 DAQ card and employed for collection and processing of measured data. A sampling rate of $1 \mathrm{KHz}$ with a total sample number of 4,000 was used to obtain smooth data. No correction has been made to the raw probe data, thus within supersonic flow regions, the normal shock wave in front of the probe tip will cause the probe to measure a reduced total pressure.

Finally, a total of 11 static pressure tappings were drilled in the aft-deck plate as shown in Fig. 4. Standard, steady state pressure transducers (type ASDX100A24R - sensitivity +/2\%) linked to a National Instruments DAQ card were employed with LabVIEW software to measure aft-deck surface static pressure; 5,000 samples with a sampling rate of $1 \mathrm{KHz}$ were used to obtain repeatable averaged pressure measurements. Data was acquired sequentially and transducer calibration was carried out in static tests using a Druck DPI610 portable pneumatic pressure calibrator.

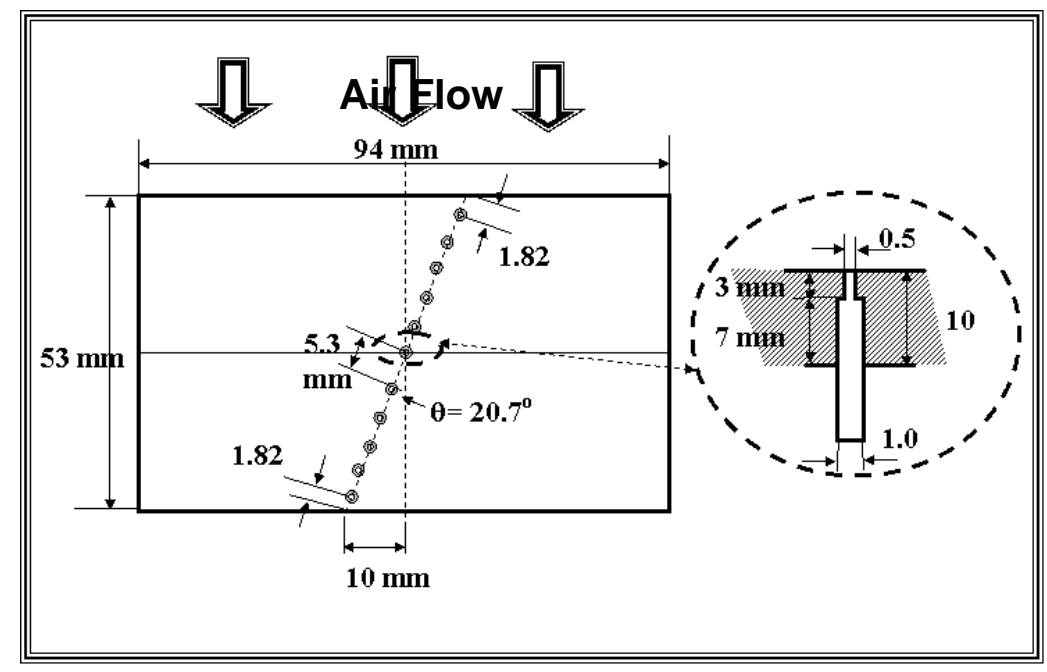

Fig. 4 Static pressure tapping positions on aft-deck

\section{Test Conditions and Datasets}

In the coordinate system shown in Fig.2, and used below to report measurements, the origin is located in the center of the nozzle exit plane; the longitudinal (x) coordinate is oriented along the jet axis (positive downstream), the transverse (z) coordinate is positive vertically upward (nozzle minor axis direction), and the spanwise (y) 
coordinate has its positive direction such that a right-handed orthogonal set is formed (nozzle major axis direction). The selected flow parameters measured were: Pitot pressure in the jet plume, static pressure on the aft-deck surface, and, using the two-component LDA: mean velocities ( $\mathrm{U}$ and $\mathrm{W}$ ), turbulent normal stress rms intensities (u-rms, wrms), and the component of the turbulent shear stress in the $\mathrm{x}-\mathrm{z}$ plane $\left(\overline{u^{\prime} w^{\prime}}\right)$.

Three operating conditions corresponding to choked, mild and moderate underexpansion (NPR=1.9, 2.45, and 3.5) were investigated in detail for both a clean nozzle and a nozzle with added aft-deck; all measurements were made without the combustor operating so that the total temperature of the jet equaled the ambient temperature for all data presented here. Several Schlieren images were taken for a range of NPRs between 1.5 and 4 and the flow structures examined before selecting the 3 NPRs noted above for detailed measurement. Total pressure and velocity profile traverses along y and $\mathrm{z}$ directions were made through the plume centerline. These cruciform profiles were measured at 15 axial stations between $\mathrm{x} / \mathrm{D}_{\mathrm{h}}=0.5$, and 10 . A total of 85 points, covering a $10 \mathrm{D}_{\mathrm{h}}$ axial distance was used for jet centerline measurements. Aft-deck wall static pressures were measured for NPRs between 1.5 and 6 . The effect of aft-deck axial length was assessed via Schlieren images for the three lengths indicated in Section IIC also at several NPRs from 1.5 to 6 . The maximum nozzle supply total pressure deviation from an operating condition set point value was $+/-1.0 \%$. The maximum measurement uncertainty estimated for Pitot pressure, mean velocity and rms data were $+/-1 \%,+/-2 \%$ and $+/-5 \%$ respectively; these uncertainty estimates are at a $95 \%$ confidence level using the recommendations given by Moffat ${ }^{27}$.

\section{Results and Discussion}

\section{A. Flow Structure Visualization}

Fig. 5 (left) presents Schlieren images of the clean jet at three NPRs: 1.9, 3.0 and 4.0. Side (minor axis) views are shown for all 3 NPRS and, for NPR=4.0 a top (major axis) view is also included. The appearance of underexpanded shock cells is clear to see for NPR>1.9, as expected for a simple convergent nozzle. The clean nozzle displays a symmetric wave structure about the $\mathrm{x}$-axis with strong expansion and compression regions, which grow in axial dimension as NPR increases; the turbulent nature of the shear layers on the edges of the central inviscid core is also clearly identifiable, growing in width with downstream distance. The top view for NPR=4 .0 
shows that although 3D effects can be identified near the jet side shear layers, the central region is two-dimensional over about $60 \%$ of its width for the first 2 cells. The appearance of more diffused shear layers in the side view for $\mathrm{NPR}=3.0$ (compared to NPR=4.0 for example) is an indication of a flapping motion induced by screech; this began at $\sim \mathrm{NPR}=2.2$, was strong for $2.45<\mathrm{NPR}<3.0$ but disappeared for NPR greater than 3.2. The NPR range over which screech occurs is related to the change in shock cell length that takes place as NPR increases. For certain lengths, the frequency of pressure disturbances created as turbulent eddies are convected through the shock cells locks on to the propagation time of the created pressure waves back towards the nozzle exit where it is then able to perturb the shear layer and reinforce the eddy strength. This feedback loop only works for certain shock cell lengths, hence the appearance/disappearance of the screech tone.
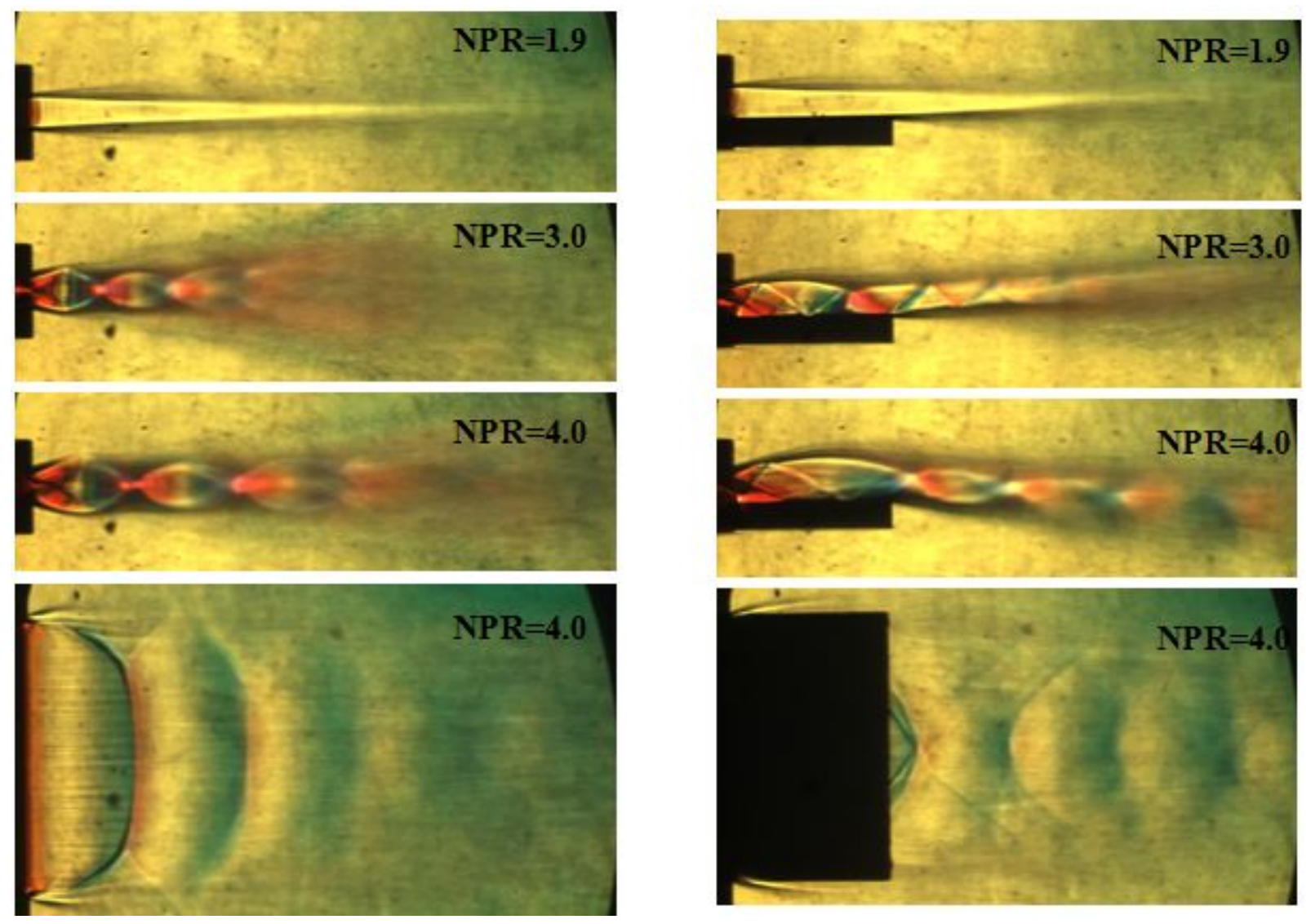

Fig. 5 Side and top views of Schlieren images of clean nozzle (left) and nozzle with datum aft-deck (right)

Fig. 5 (right) shows Schlieren images which indicate the effect on the shock structures of inclusion of the datum length aft-deck at the same three NPRs. Even at NPR=1.9 differences are visible; in this case the difference in turbulence levels generated in the upper free shear layer compared to the lower aft-deck wall boundary layer is clear 
to see. The lower shear layer, which only begins at aft-deck termination, nevertheless seems to grow more rapidly that the upper shear layer, thus the potential core region in the top image of Fig. 5 (right) is noticeably asymmetric about the x-axis. Extra reflected shocks appear with addition of an aft-deck at the higher NPRs, and the shock cell length for a given NPR increases compared to the clean case. The varying pressure force which is developed on the aft-deck wall by the shock cells (in contrast to the constant ambient pressure acting on the upper surface of the jet plume) causes a net vertical force to act on the plume, which is consequently deflected in the z-direction when it leaves the aft-deck surface. This transverse displacement of the plume can be upwards or downwards depending on the aft-deck shock structure. As NPR increases, the regions of underexpanded or overexpanded pressure lengthen on the shelf surface. The net pressure force integrated over the aft-deck surface causes the jet plume to be deflected slightly upwards at NPR=1.9 and strongly upwards at NPR=3.0; for NPR=4.0, however, the plume deflection is downwards. The pressure field on the aft-deck at NPR=4.0 is influenced by the changing shock cell pattern, but also by a separation of the plume from the aft-deck, visible in the dark region next to the aft-deck wall on the latter half of the aft-deck length. Clearly the growing wall boundary layer has been unable to sustain forward flow against the adverse pressure gradient region caused by the reflected compression wave. The NPR=4.0 top view shock structures have also changed considerably on addition of the aft-deck, showing a more 3D flow pattern. The existence of the separation region in the central portion of the aft-deck can be observed in these top view wave patterns as the flow leaves the aft-deck.

A study of the effect of aft-deck axial length on jet plume development was carried out for a range of NPR from 1.5 to 6 . Fig. 6 presents color Schlieren images of the plume over the various aft-deck lengths at NPR=4.0. The formation and size of the $1^{\text {st }}$ shock cell is effectively independent of the aft-deck length. There is clearly a strong effect of the interaction between the end of the aft-deck and the shock system, particularly when the expansion cell comes close the end of the aft-deck. For the longest aft-deck, the Schlieren image shows clear evidence of boundary layer separation and reattachment onto the aft-deck upper surface occurring for NPR=4 (Fig. 6, bottom). Note that since the net transverse pressure force exerted on the jet depends on the pressure field created on the aft-deck wall by the expansion/compression wave system, this leads to different transverse deflections of the jet plume for different lengths, upwards for the shortest and longest lengths, but downwards for the datum length. The effect of aft-deck plate trailing edge thickness on plume development was also visually investigated. Fig. 7 presents color 
Schlieren images of flow with thick and thin trailing edges at NPR=3.5. No change in plume shock structure and development was noticed at this or any other NPR tested up to NPR=6.

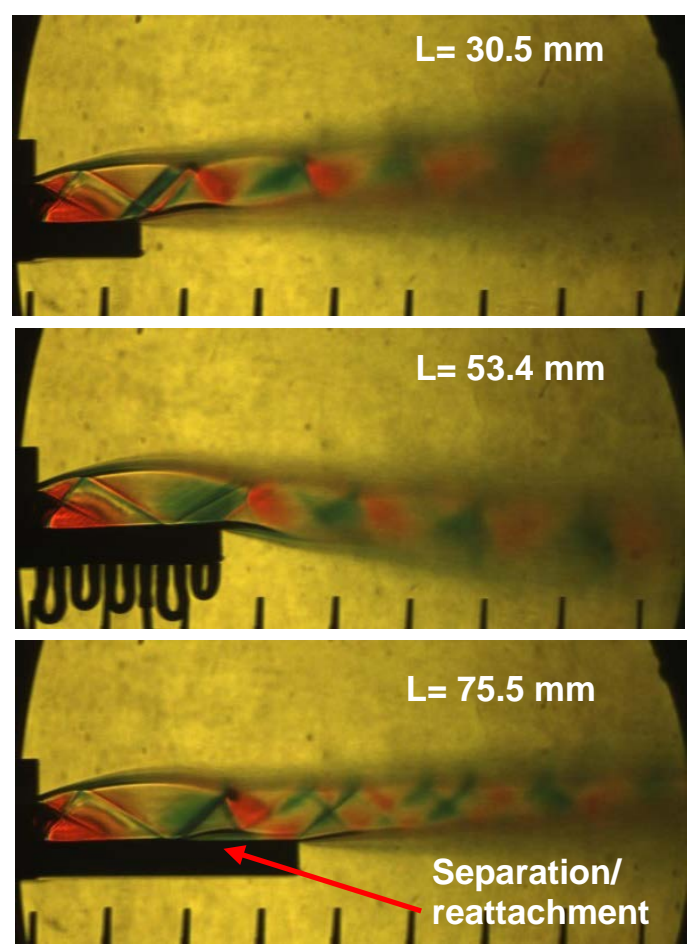

Fig. 6 Effect of nozzle aft-deck length $L$ on plume development at $\mathrm{NPR}=4.0$
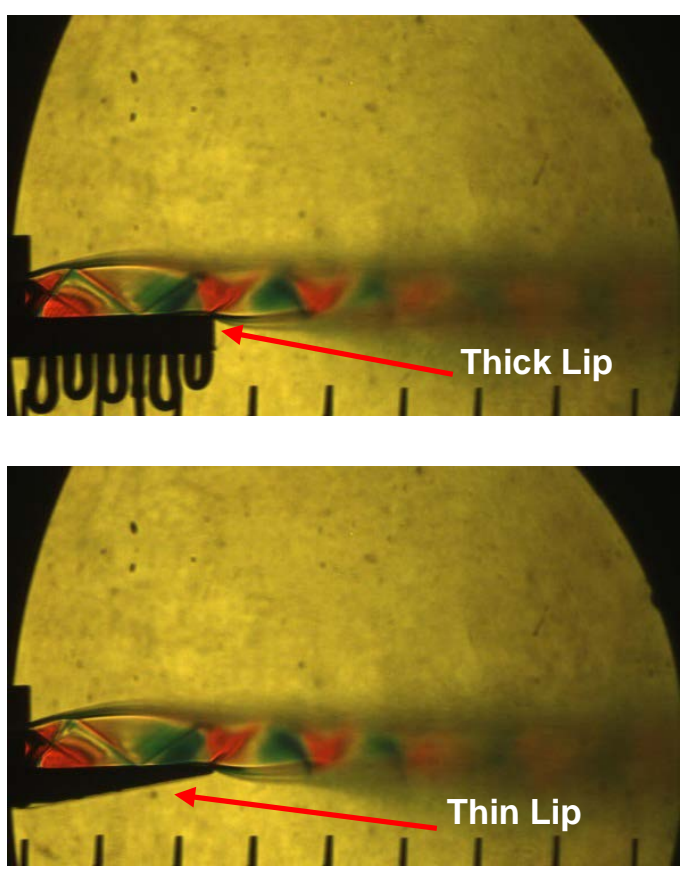

Fig. 7 Effect of aft-deck trailing edge thickness on plume development at NPR=3.5

\section{B. Static Pressure Measurements}

Fig. 8 presents measured static pressure distributions on the aft-deck for various NPRs. Unsurprisingly the static pressure only deviates noticeably from atmospheric when NPR is high enough to choke the nozzle. After this, a 


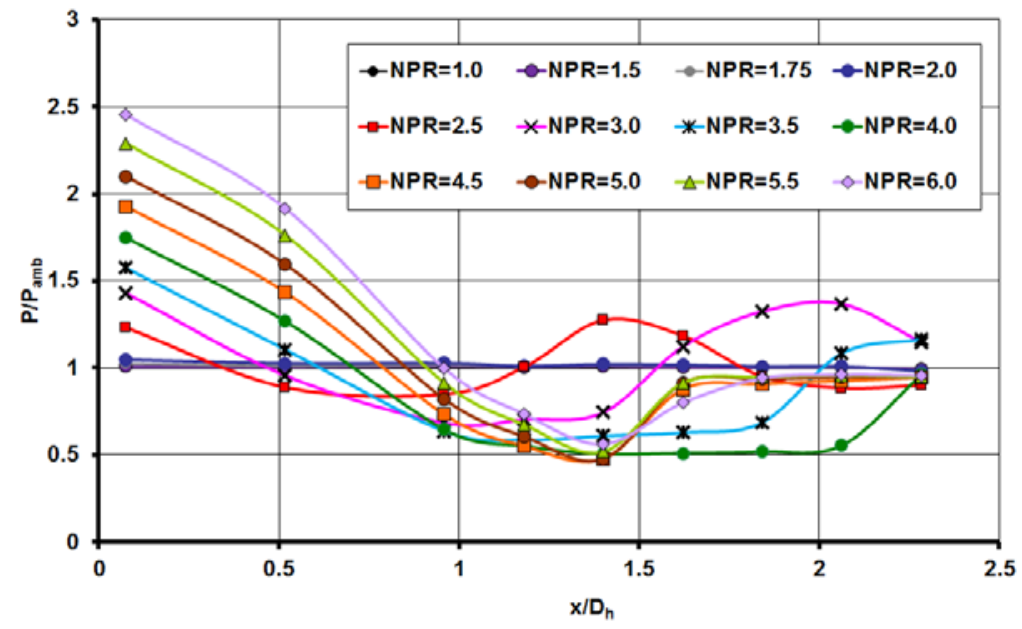

Fig. 8 Measured static pressure distribution on aft-deck

series of expansion and compression waves start to appear. As NPR increases, the strength of the first expansion region grows, and the amplitude of the adverse pressure gradient region across the reflected compression wave also increases and moves further downstream. As NPR increases beyond 3.5-4.0 the aft-deck boundary layer is unable to survive the adverse pressure gradient and a plateau in pressure over a downstream portion of the shelf is observed, indicating that separation of the aft-deck boundary layer has occurred. For NPR $>4.5$ this plateau extends to the aftdeck trailing edge, and it remains essentially the same for all NPR $>4.5$, perhaps indicating that the separation region extends beyond the aft-deck trailing edge (i.e. the closure of the separated flow region is no longer on the aft-deck upper surface, but merging with the recirculation region on the rear vertical surface of the aft-deck trailing edge has occurred and closure is in the region downstream of the trailing edge).

\section{Pitot Pressure Measurements}

Fig. 9 illustrates the development of the jet core via Pitot pressure measurements taken along the nozzle geometric centerline for all 3 NPRs and for both a clean nozzle and a nozzle with aft-deck (NB with aft-deck the nozzle geometric centerline is no longer the jet centerline). The installation of the aft-deck has little effect on jet development at the choked condition ( $\mathrm{NPR}=1.9$ ), the potential core length is unchanged at $4 \mathrm{D}_{\mathrm{h}}$, with the decay rate thereafter slightly increasing, presumably due to the extra turbulence pointed out in the lower shear layer in Fig. 5(right) for NPR=1.9. At higher NPR the potential core region contains oscillations in the Pitot pressure; these are caused because the measured pressure has not been corrected for losses across the normal shock that forms in front 
of the probe in supersonic regions. The potential core was shortened to around $3 \mathrm{D}_{\mathrm{h}}$ for the clean nozzle at NPR=2.45. This reduction was probably caused by jet instability, since for the clean nozzle as noted above, strong jet flapping due to screech was noticed between NPR 2.45 and 3.0 (see turbulence data below for supporting evidence of this). The introduction of an aft-deck stabilizes the flow and prevents flapping and the potential core length returns to $\sim 4 \mathrm{D}_{\mathrm{h}}$. In addition, it must be remembered that the plume deflection creates a non-symmetric jet profile in the minor axis (y) direction and the plume flow centerline has shifted from the nozzle geometric centerline by $\sim 0.1 \mathrm{D}_{\mathrm{h}}$ at $\mathrm{x} / \mathrm{D}_{\mathrm{h}}=3.5$. The clean nozzle plume at NPR=3.5 shows evidence of a longer inviscid potential core, with shock oscillations visible down to $\mathrm{x} / \mathrm{D}_{\mathrm{h}}=6.0$; this is an indication of increasing compressibility effects - at higher NPR the convective Mach No. $M_{c}$ across the shear layer grows and this gives rise to decreased shear layer growth rate, see Papamoschou and Roshko ${ }^{17}$ and Barone et al. ${ }^{18}$. At this higher NPR the addition of an aft-deck markedly changes the shock cell structure, both in location and also producing a more rapid decay of oscillation amplitude in the presence of an aft-deck. This is probably caused by added imperfection in the reflection of pressure waves from the thicker shear layer in the higher NPR case with aft-deck.

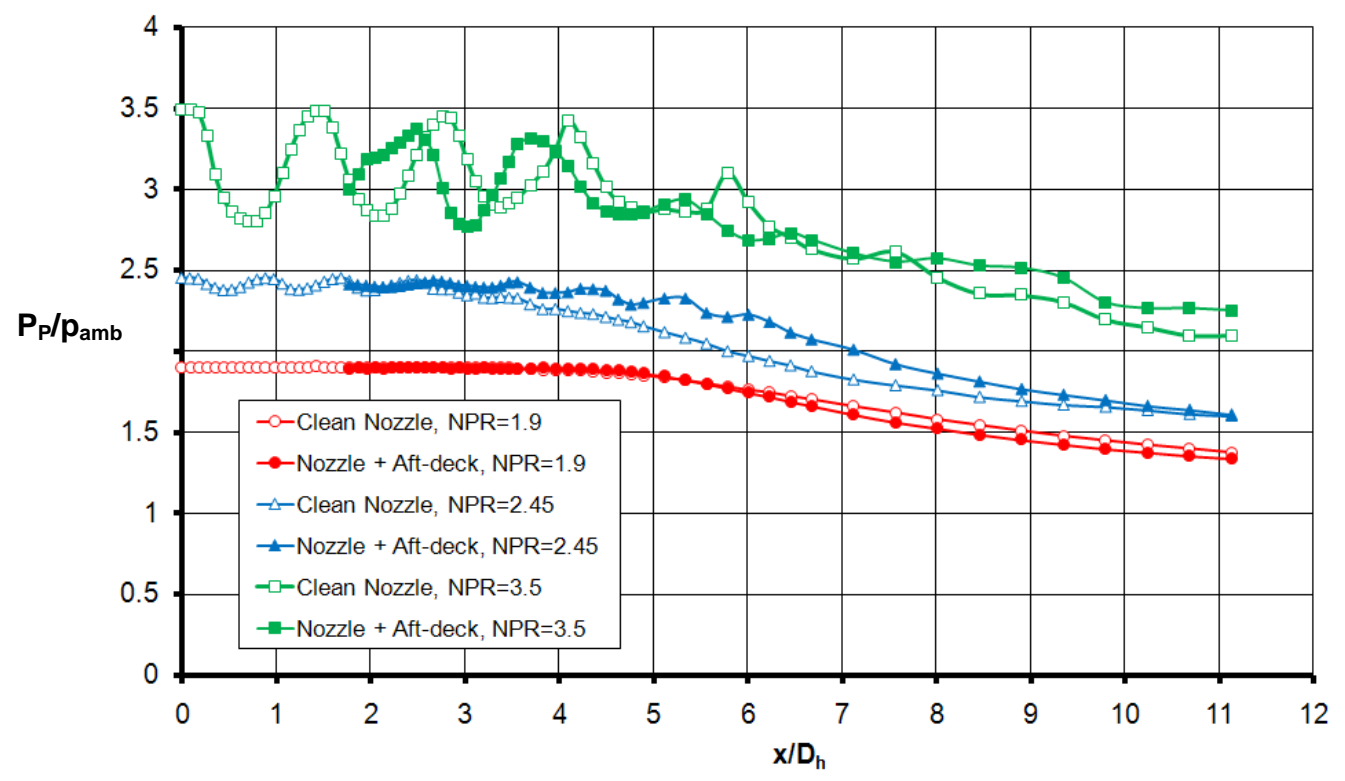

Fig. 9 Pitot pressure profiles along jet centerline at 3 NPRs

An overall first impression of plume structure for the clean nozzle may be obtained by examining measured contour maps of Pitot pressure as shown in Fig. 10 for the nozzle major (left) and minor (right) axis planes for $\mathrm{NPR}=2.45$. The very different jet core and jet edge development in these two planes is clearly visible. If the jet edge 
is characterized by a Pitot pressure $10 \%$ above ambient, then the jet cross section at $x / D_{h}=10$ is about $3 D_{h}$ in both planes, showing how much faster jet spread is taking place in the minor compared to the major axis direction. Inspection of the high $\mathrm{P}_{\mathrm{p}}$ jet core shows that in the major axis $\mathrm{z}=0$ plane, after a short region of expansion this actually contracts quite rapidly, whereas monotonic expansion is visible in the minor axis $y=0$ plane. These features are evidence of the "axis-switching” process noted in the Introduction whereby the jet cross-section, which initially has its larger dimension in the y-direction, eventually reverses this to have a larger size in the z-direction, before gradually reaching axisymmetry (the actual switch over occurs downstream of $x / D_{h}=10$ for the flow conditions shown in Fig.10).
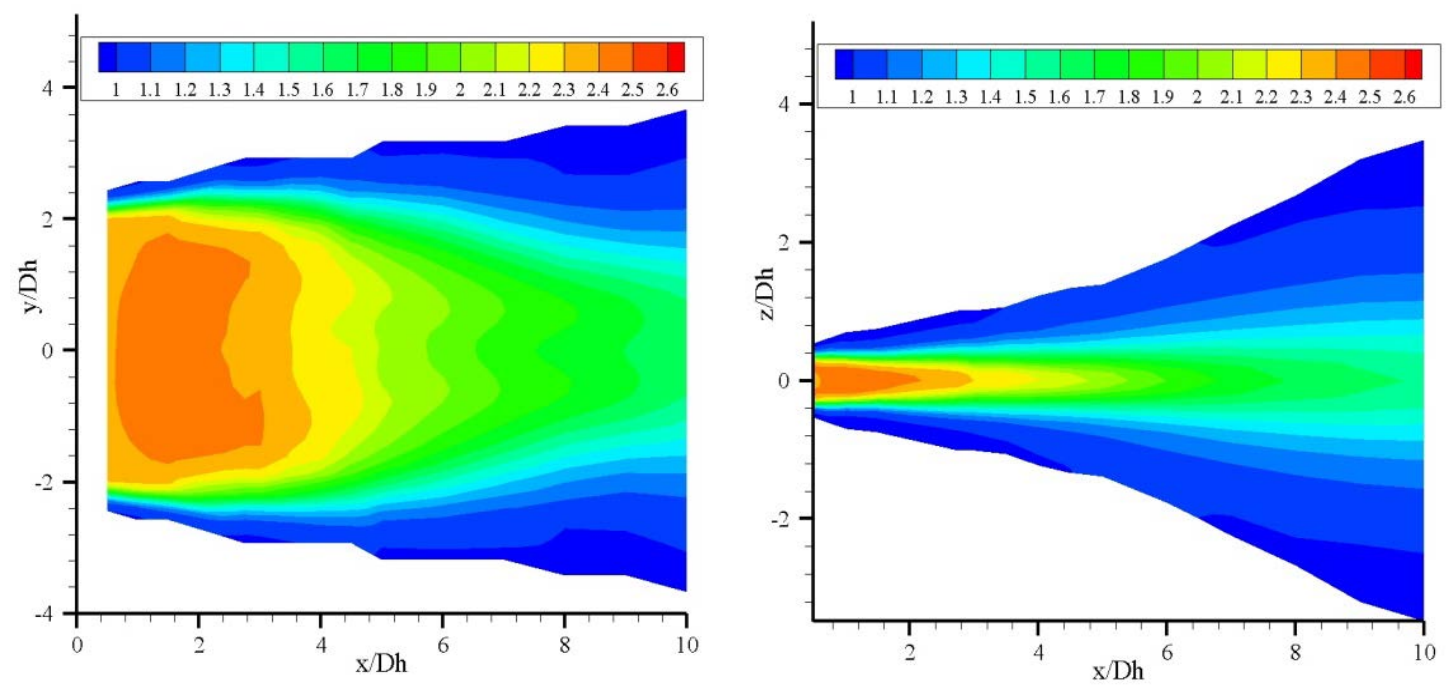

Fig. 10 Contour maps of measured Pitot pressure $P_{p}$ on nozzle major (left) and minor (right) axis planes NPR=2.45, clean nozzle

Detailed quantitative assessment of the jet plume structure is best gained from examination of y- and z-profile information. Figs. 11 and 12 present Pitot pressure profiles, again for clean and aft-deck nozzle configurations, measured on the $\mathrm{z}=0$ (left) and $\mathrm{y}=0$ (right) axes at two axial stations: $\mathrm{x} / \mathrm{D}_{\mathrm{h}}=5$ and 10 . Fig. 11 presents results for the choked case and Fig. 12 for the more strongly underexpanded operating condition NPR=3.5. The growth of the jet/ambient shear layers is easily identified from these profile measurements. For the lower NPR and a clean nozzle the shear layer growth is symmetrical in both $\mathrm{y}$ and $\mathrm{z}$ directions; in the $\mathrm{z}$ direction the shear layers have reached the axis before $\mathrm{x} / \mathrm{D}_{\mathrm{h}}=5$, but only do so in the $\mathrm{y}$ direction at around $\mathrm{x} / \mathrm{D}_{\mathrm{h}}=10$. There is only minor evidence of any 
influence of the aft-deck at this NPR at the first axial station, although, as already noted from flow visualization, a small shift in the positive $\mathrm{z}$ direction can just be identified due to the asymmetric (one-sided) entrainment in this direction. This has become much clearer by $\mathrm{x} / \mathrm{D}_{\mathrm{h}}=10$ where the jet plume centerline (peak $\mathrm{P}_{\mathrm{p}}$ location) has shifted upwards away from the nozzle geometric centerline. For NPR=3.5 (Fig. 12), the influence of the regions of supersonic flow embedded within the expansion/compression wave system as the excess jet static pressure expands back to ambient causes local regions of total pressure loss due to the intrusive nature of the pneumatic probe. For the clean nozzle this results in the 'saddle-shaped' profile at $\mathrm{x} / \mathrm{D}_{\mathrm{h}}=5$. This is much stronger in the case of the aft-deck due to the extra reflected shocks and the different development of the vortex structures caused by the aft-deck. The clean nozzle profiles remain symmetrical at both axial stations and in both y and $\mathrm{z}$ directions. The aft-deck induced change in the inviscid expansion/compression wave system, as seen in the Schlieren pictures above, leads to markedly different $\mathrm{P}_{\mathrm{p}}$ profiles, a much more pronounced double-peaked shape at the first station and a single peaked shape at the second. y-direction symmetry is preserved, but for z-direction profiles the most noticeable effect is a stronger tendency for asymmetry than at NPR=1.9, and a downwards deflection of the plume.
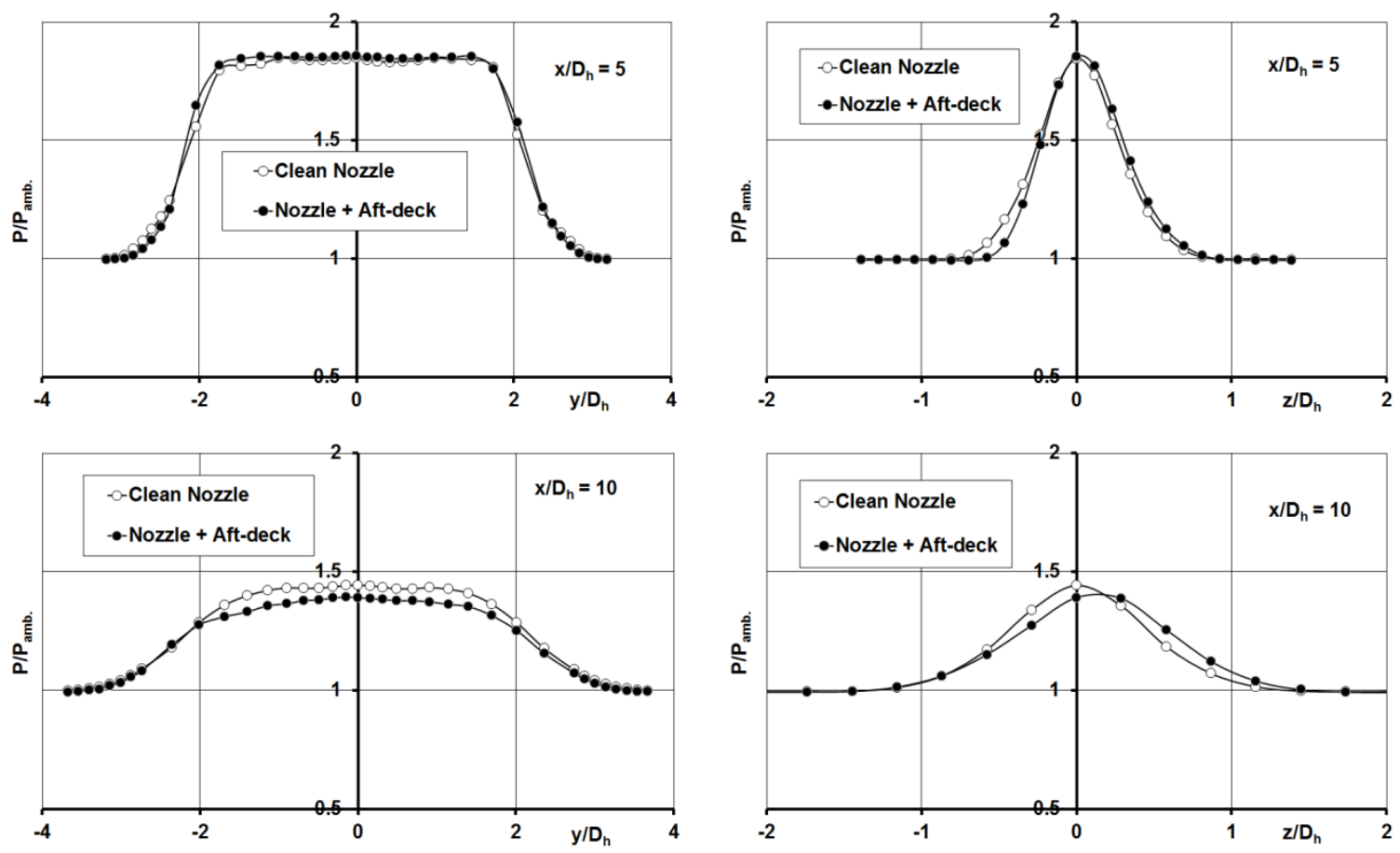

Fig. 11 Pitot pressure profiles on plume major (left) and minor (right) axes $\mathrm{x} / \mathrm{D}_{\mathrm{h}}=5$ and 10, NPR=1.9, clean nozzle and nozzle with aft-deck 

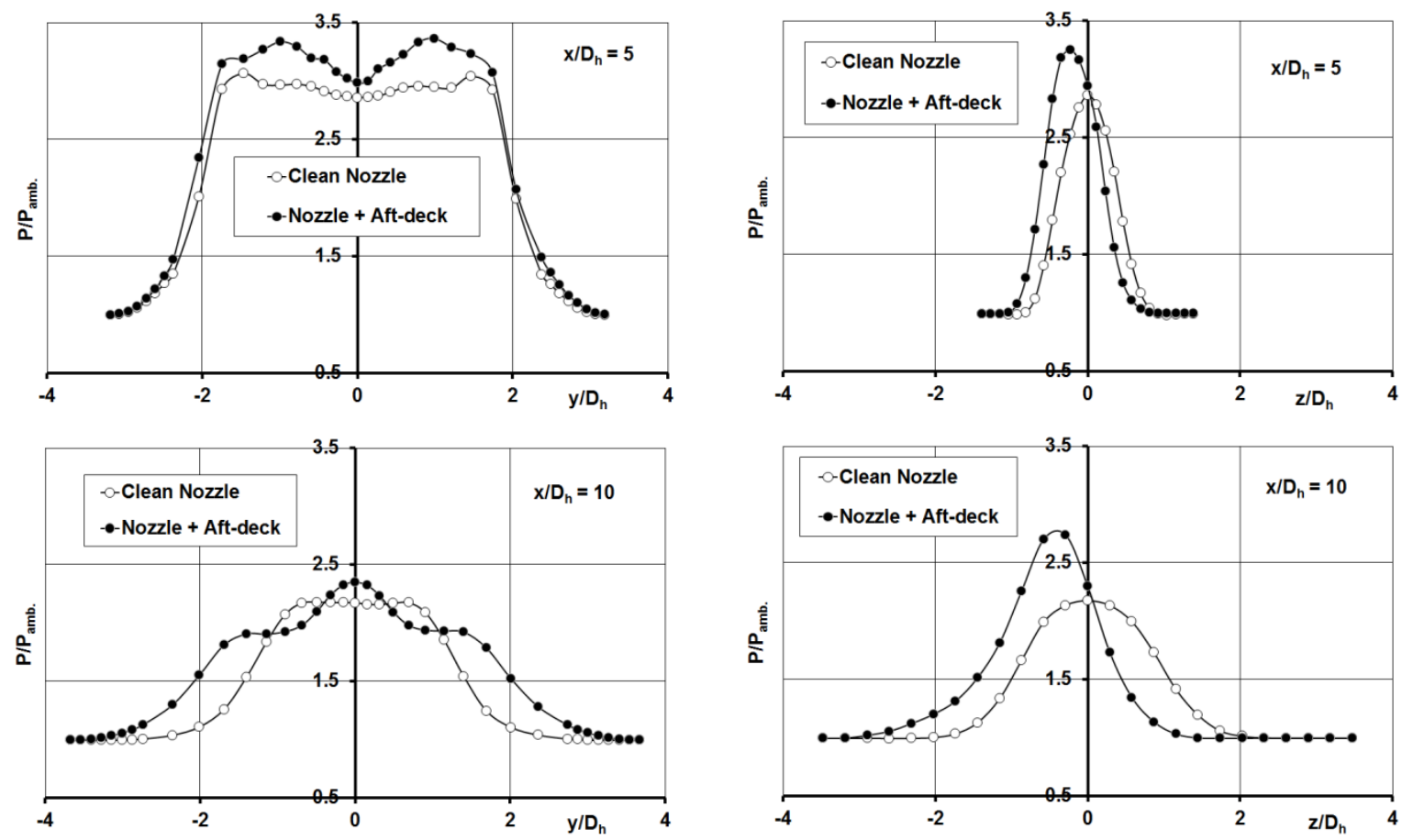

Fig. 12 Pitot pressure profiles on plume major (left) and minor (right) axes

\section{$\mathrm{x} / \mathrm{D}_{\mathrm{h}}=5$ and 10, NPR=3.5, clean nozzle and nozzle with aft-deck}

\section{LDA Measurements}

For validation of CFD predictions, for both RANS and LES turbulence closures, time-averaged mean velocity is the first basis for assessment; for judgment of turbulence modelling performance, measurement of Reynolds stress turbulence statistics is necessary. Non-dimensional contours of LDA-measured mean axial velocity $\left(U^{*}=U / U_{\text {ref }}\right)$, and two Reynolds stress fluctuating velocity normal correlations (rms intensities: $\mathrm{u}^{*}=\mathrm{u}-\mathrm{rms} / \mathrm{U}_{\text {ref }}$, $\left.\mathrm{w}^{*}=\mathrm{w}-\mathrm{rms} / \mathrm{U}_{\text {ref }}\right)$ on major and minor axis planes are used to provide an overall picture of velocity and turbulence development within the plume. This is followed by analysis of $y / z$ profiles at several axial stations on the same 
planes for better quantitative assessment; $\mathrm{U}^{*}, \mathrm{u}^{*}$, and a non-dimensional Reynolds shear stress $\left(\mathrm{uw}^{*}=\overline{u^{\prime} w^{*}} / \mathrm{U}_{\text {ref }}{ }^{2}\right)$ profiles are presented. All measured quantities have been non-dimensionalised using for $\mathrm{U}_{\text {ref }}$ the measured centerline axial velocity $(120 \mathrm{~m} / \mathrm{s})$ at the nozzle inlet plane $\left(\mathrm{x} / D_{h}=-9.1\right)$.

Fig. 13 presents contour plots of $\mathrm{U}^{*}, \mathrm{u}^{*}, \mathrm{w}^{*}$ on the minor axis plane, for a clean nozzle (left) and nozzle with aftdeck (right) and similar plots on the major axis plane are presented in Fig. 14. In order to illustrate the effect of screech, NPR=2.45 data is presented, since this was within the strong screech region. Comparing minor axis plane $U^{*}$ contours allows assessment of the effect of the aft-deck on plume characteristics in this plane, inducing a slight upward trajectory, the plume centerline shifting upwards by $\sim 0.1 D_{h}$ at $\mathrm{x} / D_{h}=10.0$. For both clean and with aft-deck flows, evidence of a saddle-shaped velocity profile in the major axis plane can be seen (Fig. 14); this, as mentioned in the Introduction, is fundamentally associated with the asymmetric vortex ring flow development observed in rectangular jets. For the clean nozzle the contour shape is influence both by the initial vortex ring appearance and also by the classical inward movement of the velocity peak in the major axis plane that is driven by the self-induced vortex velocity field. When an aft-deck is introduced, the inwards movement is suppressed, so the initial saddle shape is retained for longer downstream. This is due to different vortex dynamics for clean nozzle and aft-deck nozzles; this is unsurprising since the streamwise vortices associated with both upper and lower corners in the clean case are changed in the presence of an aft-deck to just an upper corner vortex. In general, for the clean nozzle, symmetry in both $\mathrm{y}$ and $\mathrm{z}$ planes is very good for the entire measured length, including for both turbulence components. The axial intensity displays the expected behavior, with high shear generated turbulence appearing only in the shear layers. However, anomalous behavior of the transverse intensity may be noted in the clean nozzle case on the minor axis plane (Fig.13); a transverse $\left(\mathrm{w}^{*}\right)$ intensity greater than the axial $\left(\mathrm{u}^{*}\right)$ is unexpected, as well as the maximum occurring on the centerline rather than in the shear layer. This behavior is caused by screech-induced unsteady jet flapping in the minor axis direction, which is registered by the LDA as a higher turbulence level and is most visible in the high level of vertical fluctuation on the centerline itself between $x / D_{h}=3-4$. This anomaly disappears when an aft-deck is introduced, indicating that this has suppressed the screech-induced jet flapping. 

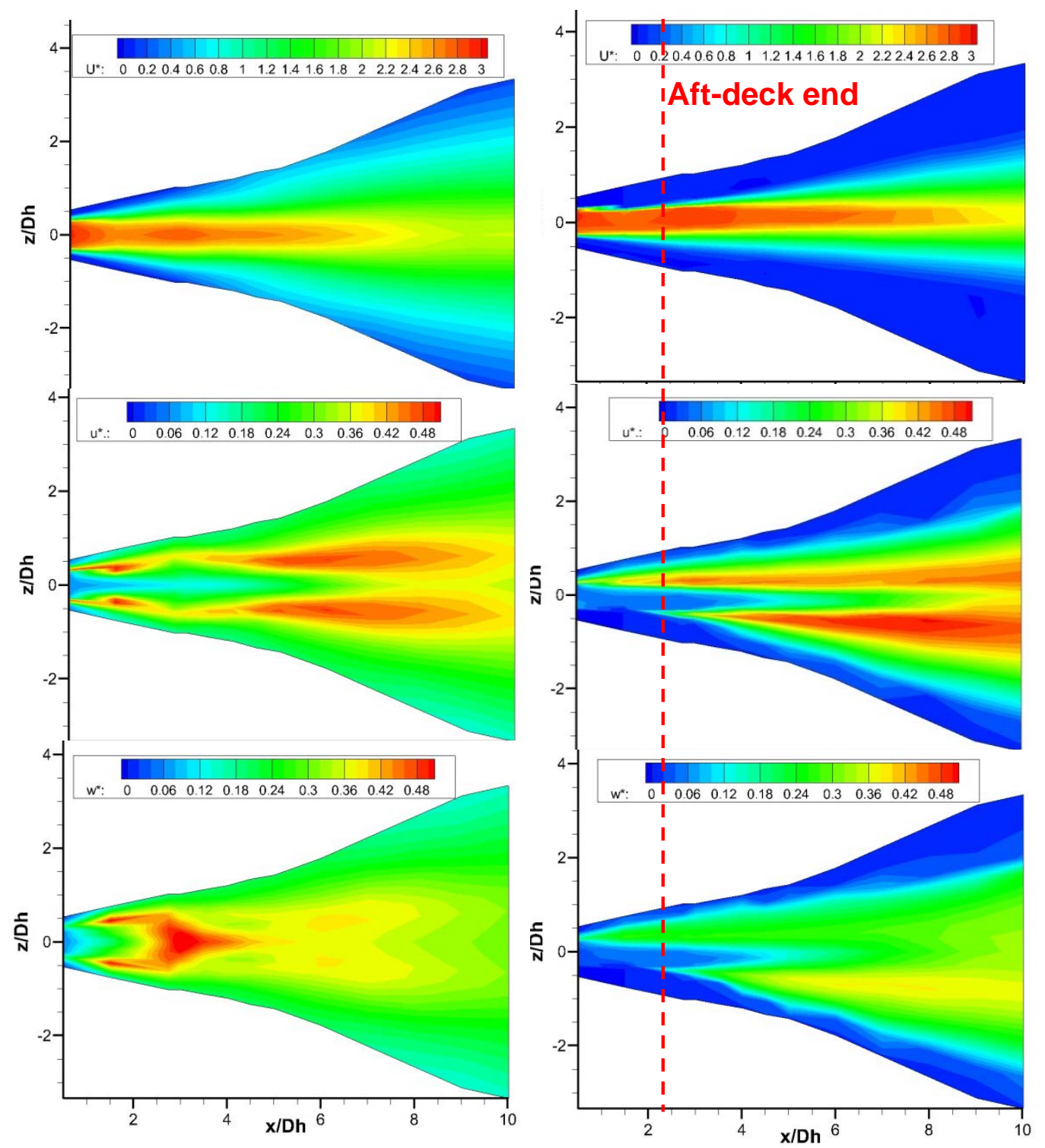

Fig. $13 \mathrm{U}^{*}, \mathrm{u}^{*}, \mathrm{w}^{*}$ contours on minor axis plane, for clean nozzle (left) and nozzle with aft-deck (right)

In Figs. 13 and 14 the z-direction asymmetry caused by the aft-deck is most obvious in the different shape of the turbulence contours in upper and lower shear layers in the x-z plane. 

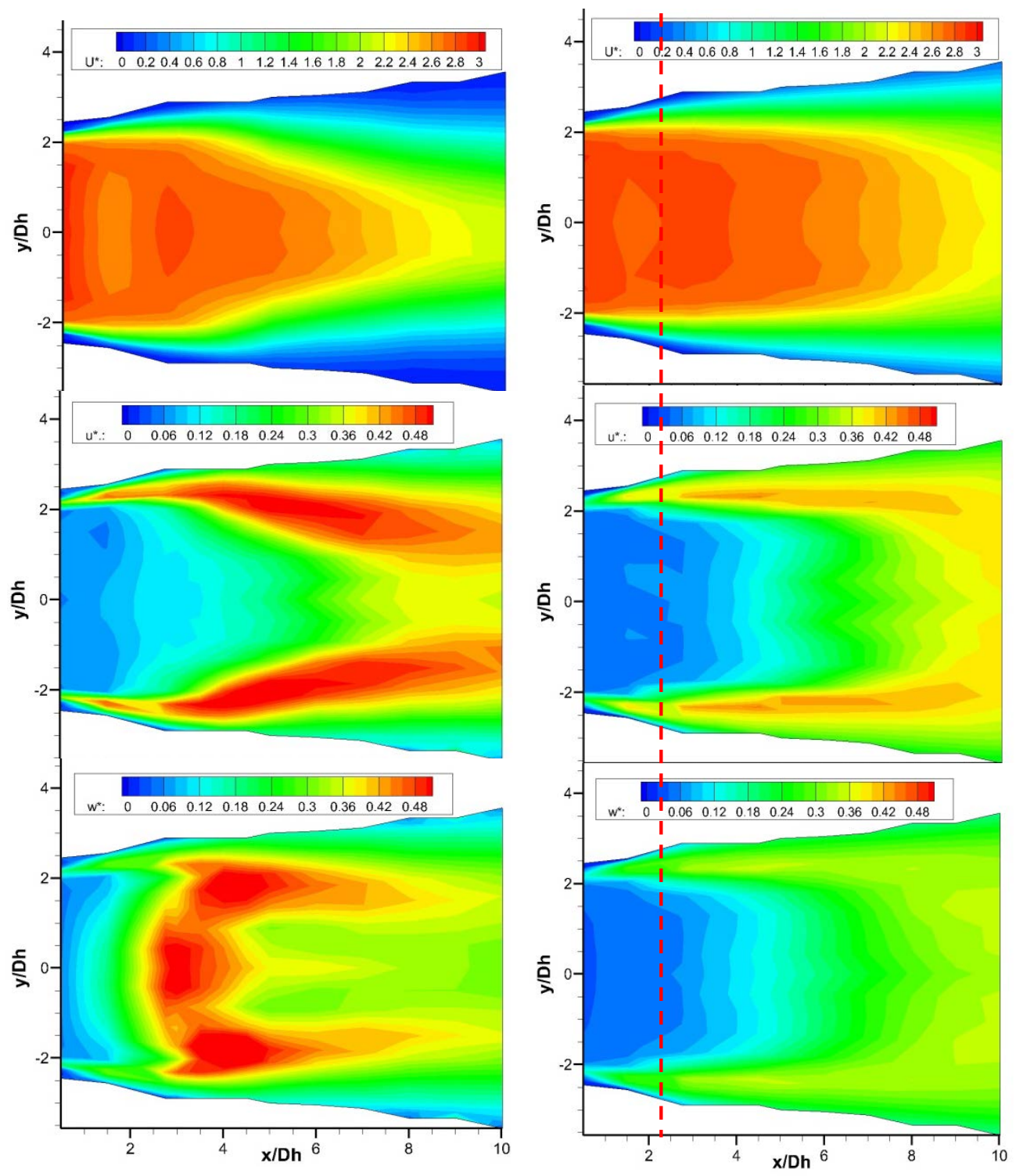

Fig. $14 \mathrm{U}^{*}, \mathrm{u}^{*}, \mathrm{w}^{*}$ contours on major axis plane, for clean nozzle (left) and nozzle with aft-deck (right)

Fig. 15 presents measured non-dimensional time-averaged axial velocity (left) and turbulence intensity profiles (right) along the nozzle centerline at NPR=2.45 for clean and aft-deck cases. As noted already from $\mathrm{P}_{\mathrm{p}}$ data, one effect of the extended shelf is a slight lengthening of the potential core length $\sim 3.0 \mathrm{D}_{\mathrm{h}}$ to $\sim 4.5 \mathrm{D}_{\mathrm{h}}$; the rate of velocity decay after the potential core is also slightly increased. Axial turbulence intensity for the clean nozzle displays the expected behavior, increasing rapidly from potential core end as the shear layers reach the centerline, and peaking a little further downstream. The measured transverse (vertical) intensity distribution is unusual. Firstly its level is 
greater than the axial data, which cannot be explained on the basis of shear generation. In a jet-like strain rate field this would produce turbulence anisotropy such that axial were stronger than transverse fluctuations. Secondly, there are 3-4 small peaks in the vertical fluctuation in the region $\mathrm{x} / D_{h}=3-4$. The explanation is again jet flapping, which will cause oscillation of the oblique shocks in the inviscid core. The turbulence anisotropy returns to the characteristics expected of shear-generated turbulence downstream of $\mathrm{x} / D_{h}=8$. The introduction of an aft-deck completely removes the anomalous turbulence behavior, consistent with the disappearance of screech at all NPRs when the aft-deck was present. With aft-deck the turbulence levels within the jet core region are initially slightly reduced compared to the clean nozzle flow, although peak values are slightly larger and occur further downstream.
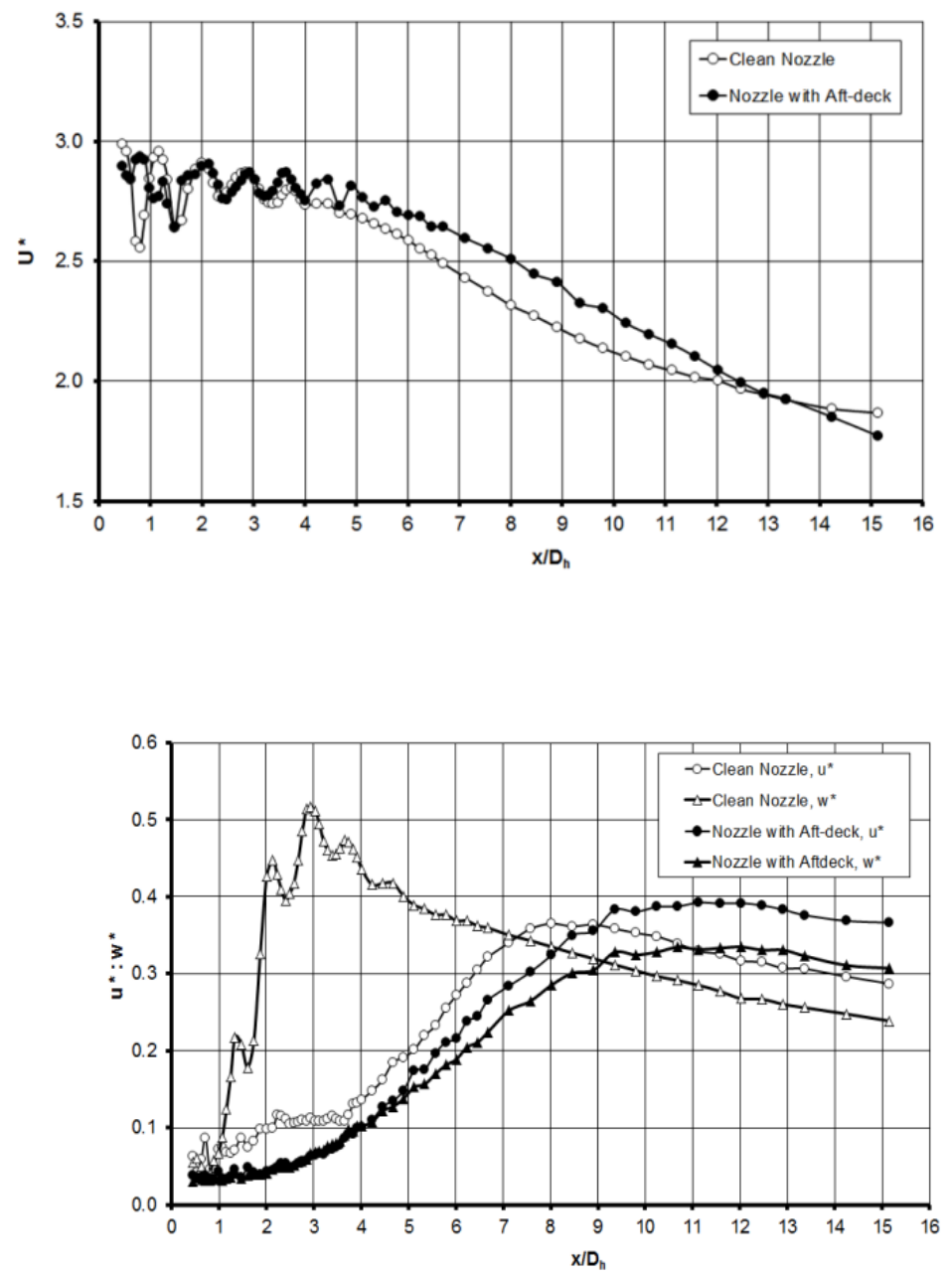

Fig. $15 \mathrm{U}^{*}$ (top) and $\mathrm{u}^{*}, \mathrm{w}^{*}$ intensities (bottom) on nozzle centerline, NPR=2.45 
Finally, to enable a more quantitative comparison than is possible on contour plots, Fig. 16 presents measured U* profiles on both minor and major axis planes, and uw $^{*}$ profiles on the nozzle minor axis plane for NPR=3.5 and for
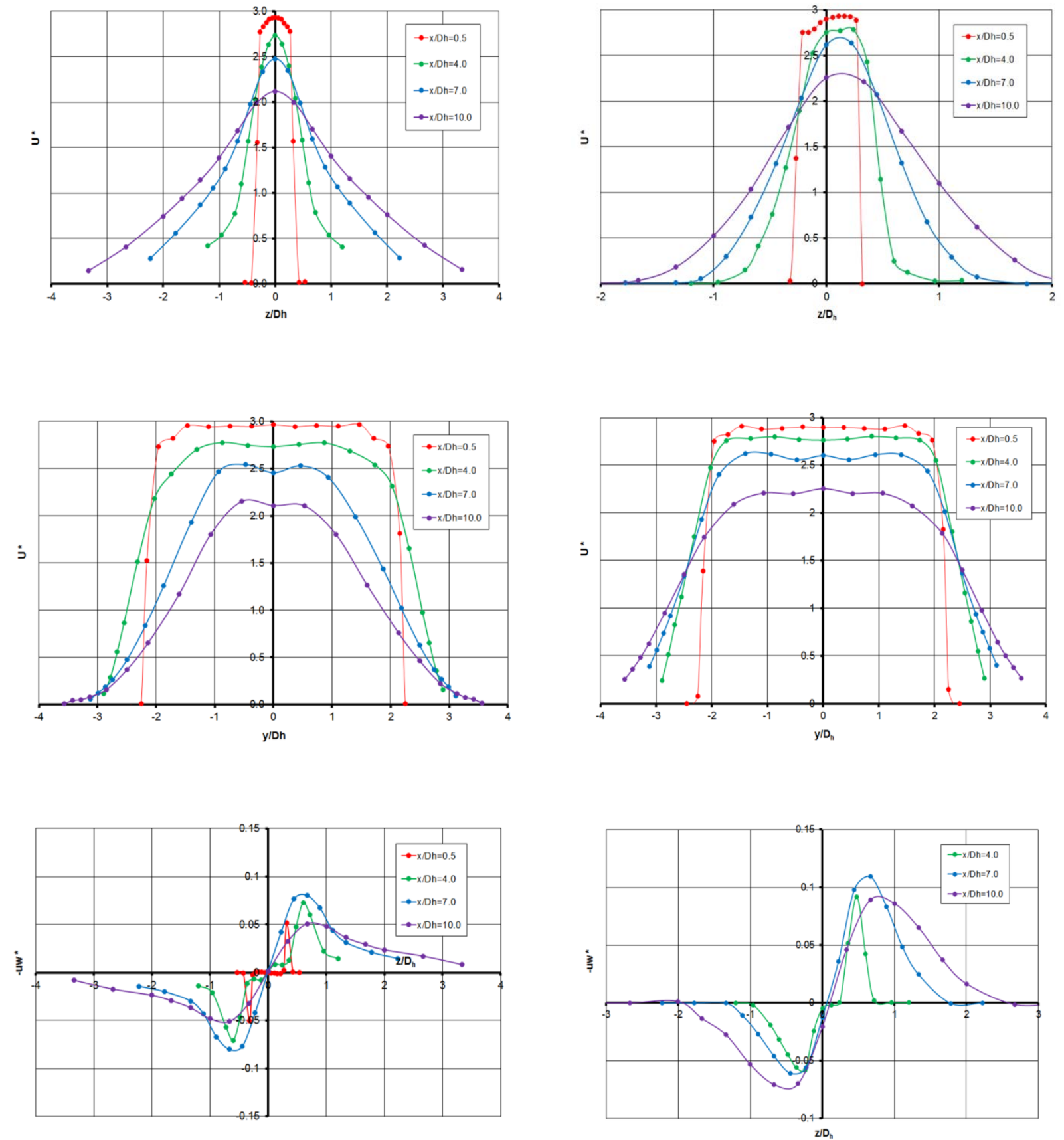

Fig. 16 Measured U* profiles on plume minor and major axes, and uw* profiles on plume minor axis, NPR=3.5 clean nozzle (left) and nozzle with aft-deck (right). 
both clean and nozzle with aft-deck. These figures provide a better appreciation of velocity gradient development in the shear layers. The effect of the z-plane asymmetry introduced by the aft-deck is also best visualized in these figures, see $\mathrm{U}^{*}$ profiles in Fig. 16 (top); enhanced early spreading in the z-direction caused by the aft-deck may be seen by comparing profiles at $\mathrm{x} / D_{h}=4.0$. Similarly, the persistence of a mild saddle-shaped profile with an aft-deck and the suppression of inward movement of the jet core are clearly seen in Fig. 16 (middle), particularly at $\mathrm{x} / D_{h}=7.0$. The cause of enhanced mixing is seen in the larger peak shear stress values seen in the aft-deck uw ${ }^{*}$ profiles in Fig. 16 (bottom); interestingly, the shear stress is not only increased on the $-\mathrm{z}$ side (aft-deck side), but also in the upper shear layer on the $+\mathrm{z}$-side. 


\section{Summary and Conclusions}

An experimental study of underexpanded jet plumes from a high aspect ratio rectangular nozzle has been described. Schlieren imaging, pneumatic Pitot probe, and non-intrusive LDA measurements were employed to capture detailed information on the flow structure and how this changed with NPR and also when a short aft-deck was attached to the lower nozzle. The effects of an extended shelf on plume development in terms of both mean flow and turbulence characteristics have been analyzed. The aft-deck creates asymmetry in the entrainment characteristics within the shear layers, and also induces a net transverse pressure force on the plume by changing the inviscid shock cell structure. This modifies the net pressure force exerted on the jet by the aft-deck surface and leads to transverse deflection of the jet, either upwards or downwards depending on NPR. For sufficiently high NPR, and a sufficiently long aft-deck, separation (and reattachment) of the plume from the aft-deck wall was observed. The experimental data for a clean nozzle and with aft-deck addition were analyzed in detail. The presence of the aft-deck had a dramatic effect on plume development, and also stabilized the flow in the NPR range where screech-induced jet flapping was observed, producing quite different turbulence fields in clean nozzle and with aft-deck flows. The addition of an aft-deck extended the potential core length slightly and reduced turbulence levels in the plume near field. Saddle-shaped velocity profiles were observed as typical of the near field of non-axisymmetric nozzles. The mean velocity and turbulence data presented here are considered of suitable quantity and quality that they form an appropriate database to act as a CFD validation test case.

\section{References}

${ }^{1}$ Trentacoste, N. \& Sforza, P. M., “Further experimental results for 3-dimensional jets”, AIAA Jnl. Vol. 5, 1967, pp 885-893

${ }^{2}$ Sfeir, A.A., “The velocity and temperature fields of rectangular jets”, International Journal of Heat Mass Transfer, Vol. 19, 1976, pp 1289-1297.

${ }^{3}$ McGuirk, J.J. \& Rodi, W., “The calculation of three-dimensional turbulent free jets”, Proc. of 1st Turbulent Shear Flows Symposium, University Park, Pa, USA, April 1977, pp 1.29-1.36.

${ }^{4}$ Krothapalli, A., Baganoff, D., and Karamcheti, K., “On the mixing of a rectangular jet”, Journal of Fluid Mechanics, Vol. 107, 1981, pp 201-220.

${ }^{5}$ Zaman, K.B.M.Q., “Axis switching and spreading of an asymmetric jet: the role of coherent structure dynamics”, Journal of Fluid Mechanics, Vol. 316, 1996, pp 1-27. 
${ }^{6}$ Ho, C-M \& Gutmark, E. J., “Vortex induction and mass entrainment in a small aspect ratio elliptic jet”, Jnl. of Fluid Mech., Vol. 179, 1987, pp 383-405.

${ }^{7}$ Hussain, F. \& Husain, H.S., "Elliptic jets, Part 1: Characteristics of unexcited and excited jets”, Journal of Fluid Mechanics, Vol.208, 1989, pp 643-668.

${ }^{8}$ Gutmark, E., J. \& Grinstein, F. F., “Flow control with non-circular jets”, Ann. Rev. of Fluid Mech., Vol. 31, 1999, pp 239-272.

${ }^{9}$ Grinstein, F., F., Gutmark, E., J., Parr, T., “Near-field dynamics of subsonic free square jets: a computational and experimental study”, Physics of Fluids, Vol. 7, 1996, pp 1483-1497.

${ }^{10}$ Grinstein, F., F., “Vortex dynamics and entrainment in rectangular free jets”, Jnl. of Fluid Mech., Vol. 437, 2001, pp 69-101.

${ }^{11}$ Lozanova, M. \& Stankov, P., “Experimental investigation on the similarity of a 3D rectangular turbulent jet”, Experiments in Fluids, Vol. 24, 1998, pp 470-478.

${ }^{12}$ Quinn, W.R., “Turbulent free jet flows issuing from sharp-edged rectangular slots: the influence of slot aspect ratio”, Experimental Thermal and Fluid Science, Vol. 5, 1992, pp 203-215.

${ }^{13}$ Quinn, W.R., “Passive near-field mixing enhancement in rectangular jet flow”, AIAA Journal, Vol. 29, 1991 , pp 515-522.

${ }^{14}$ Mi, J., Deo, R.C. and Nathan, G.J., “Characterization of turbulent jets from high aspect ratio rectangular nozzles”, Physics of Fluids, Vol. 17, 2005, pp. 068102-1 to 4.

${ }^{15}$ Krothapalli, A., Hsia, H., Baganoff, D., Karamcheti, K, “The role of screech tones in mixing of an underexpanded rectangular jet”, Journal of Sound and Vibration, Vol. 106, 1986, pp 119-143.

${ }^{16}$ Gutmark, E. J., Schadow, K. C., Wilson, K. J., “Non-circular jet dynamics supersonic combustion”, AIAA Journal of Propulsion and Power, Vol. 5, 1989, pp 529-533.

${ }^{17}$ Papamoschou, D. \& Roshko, A., “The compressible turbulent shear layer: an experimental study”, Journal of Fluid Mechanics, Vol. 197, 1988, pp 453-477.

${ }^{18}$ Barone, M. F., Oberkampf, W. L., Blottner, F. G., "Validation case study: prediction of compressible turbulent mixing layer growth rate”, AIAA Jnl., Vol. 44, 2006, pp 1488-1497.

${ }^{19}$ Feng, T. \& McGuirk, J. J., "LDA measurements in high Mach number axisymmetric jet shear layers", Proceedings of 5th International Symposium on Turbulence and Shear Flow Phenomena, Munich, August 2007.

${ }^{20}$ Deo, R.C., Nathan, G.J., Mi, J., “Comparison of turbulent jets issuing from a rectangular nozzle with and without sidewalls”, Experimental Thermal and Fluid Science, Vol. 32, 2007, pp 596-606.

${ }^{21}$ Alnahhal, M., Panidis, T.H., “The effect of sidewalls on rectangular jets”, Experimental Thermal and Fluid Science, Vol. 33, 2009, pp 838-851. 
${ }^{22}$ Alnahhal, M., Cavo, A., Romeos, A., Perrakis, K., Panidis, T. H., "Experimental investigation of the effect of endplates and sidewalls on the near field development of a smooth contraction rectangular jet”, European Journal of Mechanics B/Fluids, Vol. 30, 2011, pp 451-465.

${ }^{23}$ Lamb, M., Taylor, G., Frassinelli, M.C., "Static internal performance of a two-dimensional convergent-divergent nozzle with external shelf”, NASA Technical Memorandum 4719, 1996.

${ }^{24}$ Deere, K. A., Berrier, B. L., Flamm, J. D., Johnson, S. K., “Computational study of fluidic thrust vectoring using separation control in a nozzle”, AIAA-2003-3803, 21st AIAA Applied Aerodynamics Conference, Orlando, Florida, USA, June 2003.

${ }^{25}$ Behrouzi, P., Feng, T., McGuirk, J. J., "Active Flow Control of Jet Mixing Using Steady and Pulsed Fluid Tabs", Proc. of the I. Mech. E., Part I: Jnl. of Systems and Control Engineering, Vol. 222, 2008, pp 381-392.

${ }^{26}$ Behrouzi, P. \& McGuirk, J. J., "Effect of tabs on rectangular jet plume development", AIAA Jnl. of Propulsion and Power, Vol. 25, 2009, pp 930-939.

${ }^{27}$ Moffat, R.J., “Describing the uncertainties in experimental results”, Expt. Thermal and Fluid Science, Vol.1, 1988, pp 3-17. 